\title{
Examination of the Internal Profiles of Micro-Electrodischarge Machined Holes and the Detection of Material Defects
}

\author{
Heather Almond, David Allen and Peter Logan \\ (Received April 30th, 1999)
}

School of Industrial and Manufacturing Science, Cranfield University, Bedford, UK

\begin{abstract}
Micro-electrodischarge machining (micro-EDM) is used to machine micro-features such as holes, slots and cavities in electrically conductive materials. This paper looks at the fabrication of micro-holes for ink jet printing nozzles where hole diameters are smaller than $50 \mu \mathrm{m}$. It is important that the printing medium be able to flow through unimpeded, so the bore must be as smooth as possible. However, it is difficult to inspect the internal profiles of such small features non-destructively. In this study, micro-holes were machined along the join between two adjacent foils to provide a natural means of separation without using a secondary process to cut the holes in half. It was then possible to investigate the holes and unmachined control samples using scanning electron microscopy (SEM). It was found that defects such as micro-cavities, cracks and inclusions were already present prior to machining by micro-EDM, seemingly as a result of the material rolling process.

Key words: micro-electrodischarge machining, micro-EDM, micro-holes, internal profiles
\end{abstract}

\section{INTRODUCTION}

Micro-EDM is a non-contact erosion process where frequent energy discharges between a charged tool electrode and an oppositely charged conductive workpiece give rise to material removal from both workpiece and tool. This is achieved in an essentially burr free manner. The frequency of the discharges gives rise to the characteristic microEDMed surface finish; tiny overlapping craters (Fig.1). Surface roughness values of less than $0.2 \mu \mathrm{m}$ have been recorded ${ }^{1), 2}$.

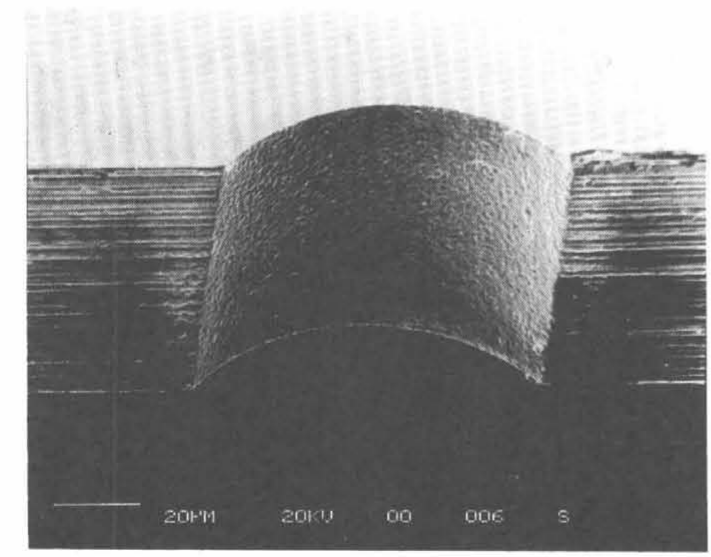

Fig.1 Typical micro-EDMed surface finish

The process is typically used to machine holes, slots and cavities for applications such as micro-orifices for the medical and aerospace industries, micro- filters, devices for medical implants, electron beam gun apertures, micro-dies and printing devices.

The micro-EDM process offers two major advantages; the ability to achieve high aspect ratios and the ability to machine conductive materials irrespective of their hardness. Aspect ratios of greater than 10 are possible. This is particularly important in fluid-related applications, where certain flow or guiding characteristics are required ${ }^{3}$. Where the fluid medium may be abrasive or corrosive it is often necessary to achieve these higher aspect ratios in hard "difficult-to-machine" materials such as stainless steels.

In the fabrication of industrial ink jet printers, the quality of drop formation and the directional stability of the jet govern the printer performance. Both of these features are primarily controlled by the nozzle characteristics ${ }^{4}$. (In this study the nozzles consisted of a line of micro-holes machined in a metal foil). So, in addition to an aspect ratio of, say 2.5 , in a hard material like stainless steel, a smooth bore must be machined such that it will not impede the trajectory of the ink jet and give rise to unwanted displacement of the ink during printing. Micro-EDM is one of the few machining techniques that is able meet these stringent requirements. An average surface roughness of $187 \mathrm{~nm}$ has been measured by Atomic Force Microscopy (AFM) for the bore surface ${ }^{2)}$. 
High aspect ratios obviously make "inside hole" inspection for inclusions, cavities and any other features that may affect jet performance very difficult. A nozzle's performance can only be tested after manufacture and this will indicate whether it is successful or a reject and indeed which jets (or micro-holes) are problematical. However, it will give very little information about the causes of any irregularities, as the defects are not visible.

It was with this latter point in mind that this study was carried out. Previous investigations using nondestructive methods such as X-ray micro focusing to detect defects in the bore of ink jet nozzles had proved unsuccessful. It was decided that a simple alternative would be to micro-EDM a line of holes between two adjacent nozzle foils and inspect the separate halves afterwards. This would eliminate the need to use a secondary process to cut the holes in half. (Feasibly, a secondary process could cause mechanical damage, which might mask any irregularities created by the machining process). Thus, having overcome the aspect ratio problem, it would then be possible to investigate the holes by SEM and assess the influence of the micro-EDM process on the smoothness of the bore.

\section{THE MICRO-EDM PROCESS}

The micro-EDM process works in the same manner as conventional EDM. A cathode and anode (the workpiece) are situated in a dielectric medium. An applied voltage causes sparking across the cathodeanode gap. The resultant ionisation of the dielectric causes material to melt and vaporise from both electrodes. The local temperature will be of the order of $10,000 \mathrm{~K}$ and the discharge energy is about $10^{-7} \mathrm{~J}$. Each discharge follows the path of least resistance i.e. the point at which the anode and cathode are closest. Debris from the process is flushed away by the dielectric. The gap distance is maintained at $5-10 \mu \mathrm{m}^{5)}$ by the servomechanism to produce a constant erosion process. In this way, the workpiece is eroded such that it replicates the reverse shape of the tool electrode.

The fabrication of nozzles for high-speed ink jet printheads is a relatively specialised process. In addition to the requirement to achieve high aspect ratio micro-holes in hard (non-corrosive/abrasionresistant) materials with a smooth bore, high positioning accuracy $( \pm 0.5 \mu \mathrm{m})$ and repeatability in the machining of individual holes is also required. Consequently, there are not many commercial systems available. The work detailed in this paper was carried out using one particular research machine. However, the same operational parameters were used as an identical production machine. In addition, the same effects as described a little later had been observed by another operator. This indicates that the results obtained were probably operator and parameter independent.

The micro-EDM system used in this work consists of two NC machines, a Charmilles EL-10 and HO10. The former is used for making a tool electrode by the Wire Electro Discharge Grinding (WEDG) process which reduces the diameter of a tungsten or tungsten alloy wire blank. This electrode is then removed (supported by its encasing mandrel) and repositioned on the HO-10 and used to die-sink the corresponding cavity into the workpiece. On both the EL-10 and HO-10, machining takes place within a dielectric medium, a hydrocarbon oil. Fig.2 demonstrates the two processes.

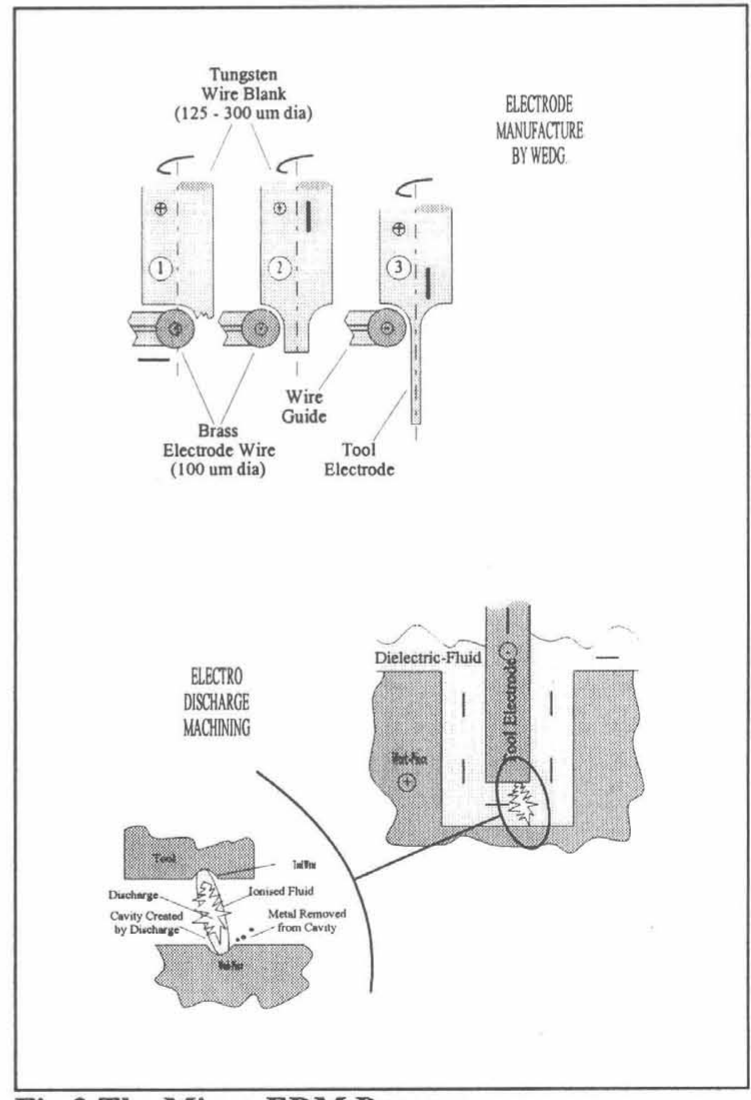

Fig.2 The Micro-EDM Process

\subsection{Electrode fabrication by micro-WEDG}

The tool electrode is the reverse shape of the required cavity to be machined in the workpiece. So for a hole it will take the form of a wire blank. A typical starting diameter for the wire blank is $125 \mu \mathrm{m}$. This is mounted vertically and connected into the circuit as the anode. It is spun at $3000 \mathrm{rpm}$. A continuously fed brass wire (the cathode) with a diameter of $100 \mu \mathrm{m}$ is fed in horizontally towards the 
rotating anode. Simultaneously, the anode is cycled in the vertical plane. The net effect of these actions is the diameter-reduction of the blank.

\subsection{Micro-EDMing a hole}

The tool electrode (cathode) is mounted above the workpiece (anode) and spun at $3000 \mathrm{rpm}$. The electrode is capable of cycling in the vertical plane and the workpiece, in the $\mathrm{X}$ and $\mathrm{Y}$ axes. The combination of these movements results in the rotating electrode being lowered into the workpiece to the required depth to create a hole and then retracted up above the surface. It is then moved along by the necessary pitch and the process repeated to complete an array.

\section{EXPERIMENTAL PROCEDURE FOR THE INSPECTION OF THE INTERNAL HOLE PROFILES}

The original idea behind the experimental work was to observe the effect of micro-EDM on the internal profile of micro-holes. There was evidence to believe that individual jets could be impeded in some way such that the trajectory of the ink passing through would become unacceptable. Possible causes included a blockage by a piece of carbonaceous by-product from the micro-EDM process or aberrations in the bore wall itself. An inspection process that would not cause additional damage to the micro-holes was required.

The following concept was developed. Machining along the join between two adjacently mounted foils would closely simulate the original machining situation The two halves could be easily separated to enable investigation of the bore. Fig. 3 shows an array of "half-holes" along the edge of a foil of stainless steel, AISI 316LN. (These particular holes were over-sized and were of the order of $\varnothing 100 \mu \mathrm{m})$.

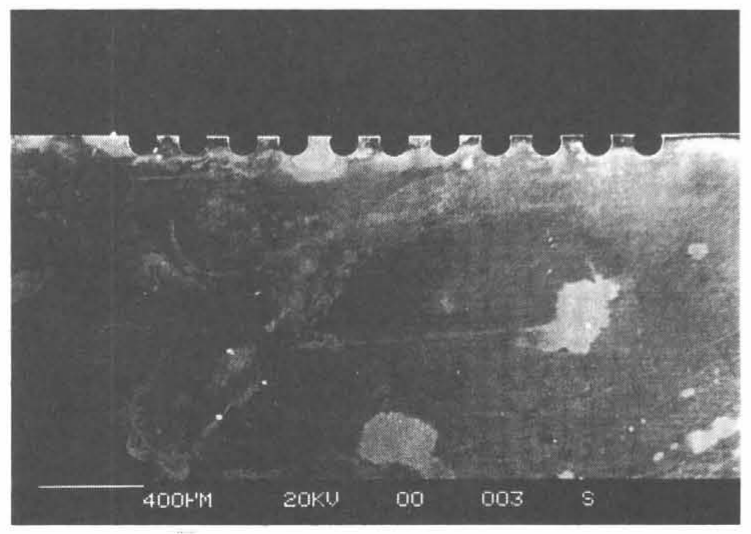

Fig.3 An array of "half" micro-holes, microEDMed along the join between two adjacent foils
A preliminary experiment was carried out using stainless steel (AISI $316 \mathrm{LN}$ ) to gain familiarity with the method. Having machined and observed the sample under SEM, several considerably sized defects (i.e. $2-10 \mu \mathrm{m})$, namely cracks and inclusions, were observed (Fig.4). The cracks were always in the rolling direction of the material indicating that the defects could be material-dependant rather than machining-dependant.

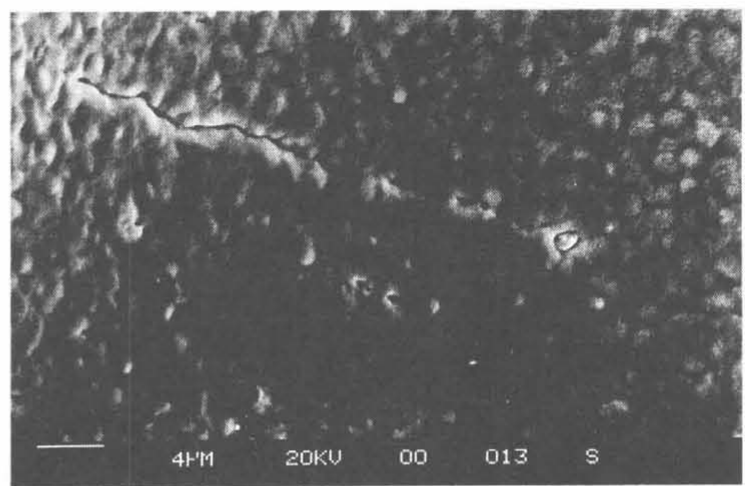

Fig.4 A crack and inclusion observed in one of the micro-holes (AISI 316 LN)

It was decided that in order to be able to attribute the defects to the micro-EDM process or the raw material state unmachined samples should be used as a control. The unmachined samples were prepared and inspected in the same way as the micro-EDMed ones.

Six metals were selected for investigation. These were all corrosion resistant materials and included four types of stainless steel and Titanium and Nickel-titanium (Table 1). The thickness of each foil is given.

\begin{tabular}{|c|c|c|c|c|c|c|c|c|c|}
\hline \multirow{3}{*}{$\begin{array}{l}\text { Material } \\
\text { stainless stee }\end{array}$} & \multicolumn{8}{|c|}{ Composition (\%) } & \multirow{2}{*}{$\begin{array}{l}\text { Thick } \\
\text {-ness } \\
(\mu \mathrm{m})\end{array}$} \\
\hline & C & Si & Mn & $\mathbf{P}$ & $\mathbf{S}$ & $\mathrm{Cr}$ & Mo & $\mathrm{Ni}$ & \\
\hline & \\
\hline AISI 316 LN & 0.03 & 0.75 & 2.000 & 0.05 & 0.03 & $\begin{array}{l}16.0 \\
18.0\end{array}$ & $\begin{array}{l}2.0 \\
3.0\end{array}$ & $\begin{array}{l}\ln 00 \\
14.0\end{array}$ & 91 \\
\hline AISI 316 LS & 0.03 & 0.75 & 2.00 & 0.04 & 0.02 & $\begin{array}{l}160 \\
18.5\end{array}$ & $\begin{array}{l}2.00 \\
2.30\end{array}$ & $\begin{array}{l}11.0 \\
13.0\end{array}$ & 137 \\
\hline BS $316 \mathrm{~S} 13$ & 0.03 & 1.060 & 2.00 & 0.05 & 0.03 & 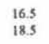 & $\begin{array}{l}2.50 \\
3.00\end{array}$ & $\begin{array}{l}11.5 \\
15.5\end{array}$ & 113 \\
\hline AISI 304 & 0.08 & 1.00 & 2.00 & 0.05 & 0.03 & $\begin{array}{l}18.0 \\
20.0\end{array}$ & . & $\begin{array}{l}8.00 \\
10.5\end{array}$ & 70 \\
\hline \multicolumn{10}{|c|}{ non-ferrous materials } \\
\hline \multicolumn{3}{|c|}{$\overline{\mathrm{Ti}}$} & \multicolumn{6}{|c|}{$99.89 \%$ pure $\mathrm{Ti}$} & 129 \\
\hline \multicolumn{3}{|l|}{ NiTi } & \multicolumn{6}{|c|}{$50 \% \mathrm{Ni}$ and $50 \% \mathrm{Ti}$} & 260 \\
\hline
\end{tabular}

Table 1. Foil materials used in study

\section{i) Preparation of the control samples}

Sample foils were mounted "edge-on" and potted in Bakelite. They were ground with 1200 grit and diamond polished $(6 \mu \mathrm{m}$ and $1 \mu \mathrm{m})$. Grinding and 
polishing was unidirectional, across the thickness of the foil. This was in an attempt to avoid inducing cracks in the rolling direction. The samples were then ready for inspection by SEM.

\section{ii) Preparation of micro-EDMed samples}

The foil samples were held in pairs in a special fixture for unidirectional grinding and polishing as for the control samples. In addition to providing a good surface finish for inspection, this also meant that the foil pairs would mate together better in preparation for machining. The foil pairs were mounted side by side on a machining jig using a conductive silver paste. The jig was then positioned on the bed of the Charmilles HO-10 and holes of less than $\varnothing 50 \mu \mathrm{m}$ were micro-EDMed along the join through the thickness of the foils. The machining conditions were as follows:

$\begin{array}{ll}\text { Voltage: } & 70 \mathrm{~V} \\ \text { Capacitance: } & 10 \mathrm{pF} \\ \text { Machining speed: } & 1.5 \mu / \mathrm{s} \\ \text { Spindle speed: } & 3000 \mathrm{rpm}\end{array}$

Machining was carried out in the controlled environment of a cleanroom (class 1000).

At least 20 holes were machined per sample. Periodic adjustment of the machining position was necessary to keep the holes aligned to the join. After machining the foils were detached from the jig and underwent cleaning to remove the silver paste and debris from the micro-EDM process.

Mechanical cleaning, using a magnetic stirrer and follower with the foils suspended from above, was used rather than ultrasonic cleaning to avoid the possibility of inducing cavitation in the foils. A $2 \%$ $(\mathrm{v} / \mathrm{v})$ Micro-90 solution was used. It was subsequently found that this method was not sufficient to remove all the silver paste residue and had the effect of partially dissolving it and then redistributing the fine sediment over a larger surface area. In addition, it did not unblock debris from all the holes. Even though the semi-holes were completely open to the cleaning fluid, at less than $\varnothing 50 \mu \mathrm{m}$, access into the curvature was still difficult.

The micro-holes then underwent inspection by SEM. All machined holes were studied and micrographs taken of the most obvious defects.

\section{RESULTS}

The main finding was that defects occurred on both the unmachined (control) and machined samples in the same abundance. This indicates that most of the defects were already present in the raw material prior to the machining process.

\subsection{The control samples}

The stainless steel samples all showed some form of irregularity to varying degrees. These were generally in the form of holes, cracks and inclusions. The AISI 316 LS sample was the best and BS 316 S13 the worst in terms of the number of apparent defects. Holes and inclusions were of the order of $\varnothing 1-2 \mu \mathrm{m}$ and cracks of $10 \mu \mathrm{m}$ and even larger were observed. Fig. 5 shows a micrograph of the unmachined AISI $316 \mathrm{LN}$ sample. This shows a hole and a crack.

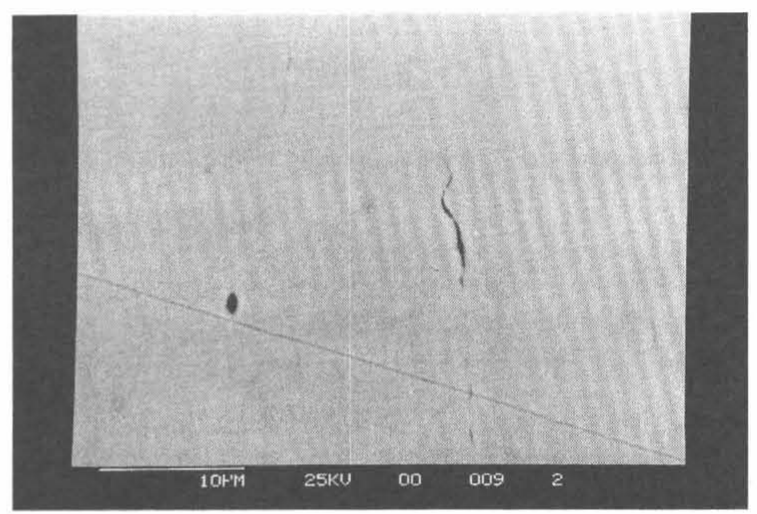

Fig.5 Unmachined AISI 316 LN, showing a hole, a crack and a polishing scratch

An inclusion on the AISI $316 \mathrm{LN}$ sample (Fig.6) was shown to contain $\mathrm{Mn}$ and $\mathrm{Cr}$ (in greater quantities than the bulk material) and Ti. A very feint polishing line runs horizontally across the middle of the micrograph through the diamondshaped inclusion.

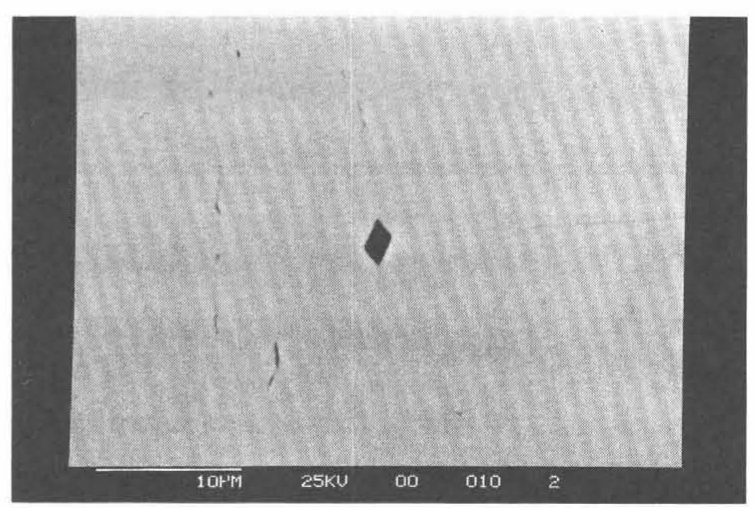

Fig.6 Inclusion in AISI 316 LN, small cracks and very feint polishing line (perpendicular to the rolling direction)

Fig.7 shows an example of the type of defect found in the AISI 304 sample. Again, the crack runs in the rolling direction. 


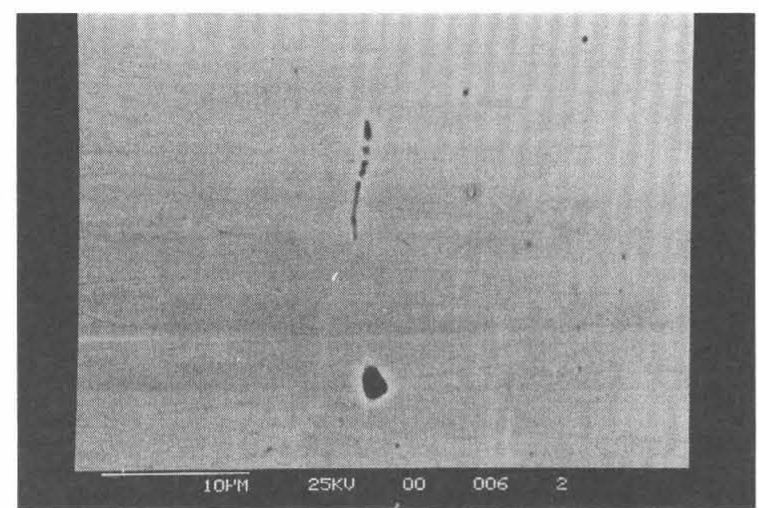

Fig.7 Defects in unmachined AISI 304

The Ti sample proved to be practically blemish-free, whereas the NiTi sample showed many holes (or inclusions) but no major cracks. An elemental analysis of an inclusion in the NiTi sample indicated that it contained more $\mathrm{Ti}$ than the matrix material.

A significant point to make is that all cracks observed in the control samples occurred in the rolling direction of the material i.e. perpendicular to the grinding/polishing direction.

\subsection{The micro-EDMed samples}

SEM of the machined samples produced similar results to the unmachined control samples. The BS 316 S13 sample showed the most defects. Fig. 8 shows a crack running through a micro-hole. This measures at least $15 \mu \mathrm{m}$ (it is a possibility that the crack extends further beyond the curvature of the micro-hole into the bulk material). It is highly feasible that a crack of this size located in a microhole would have some affect on the flow of fluid passing through.

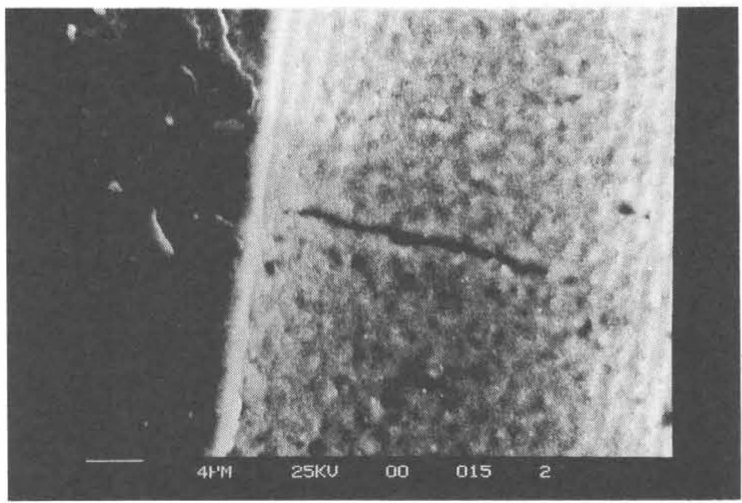

Fig.8 Crack in micro-hole, EDMed in BS 316 S13

Similar sized cracks were found in AISI $316 \mathrm{LN}$ but to a much lesser extent (one of two cracks occurring over 20 micro-holes). An example is given in Fig.9. It should be noted that there are large particles of debris still affixed to the sample i.e. the sample is relatively dirty as will have been apparent in some of the other micrographs shown here. As explained previously, ultrasonic cleaning was avoided to eliminate the possibility of cavitation. Again, both of the features shown are fairly large at $10-20 \mu \mathrm{m}$.

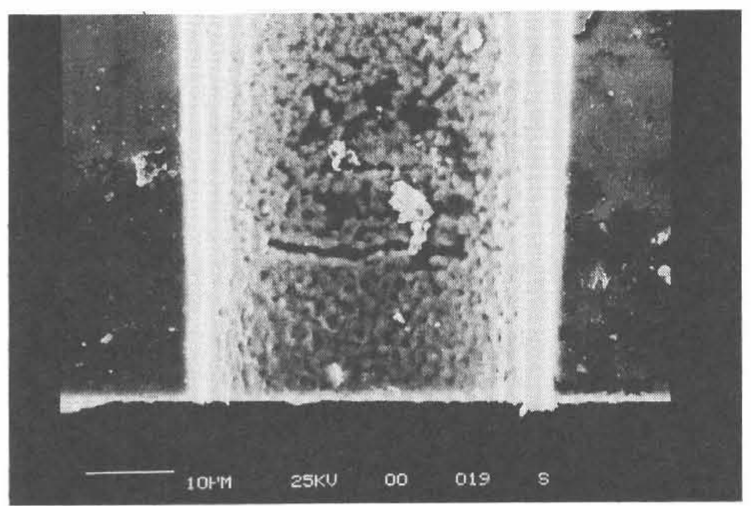

Fig.9 Cracks in micro-hole, EDMed AISI 316 LN

Preliminary familiarisation using AISI 316 LN (machining over 100 micro-holes) had resulted in the same effects. A different operator using the same research machine prior to this particular research had also observed the same features. (Here, an ink jet array had been laser-cut in half and studied using SEM. However, it had been difficult to attribute the cause of the cracks observed, as they could have been laser-induced).

The AISI 316 LS showed very few defects with no obvious cracks and only one possible cavity of about $\varnothing 2 \mu \mathrm{m}$ over the 20 micro-holes inspected.

The AISI 304 sample was still very dirty after cleaning but showed the same trends as the control samples i.e. a few minor holes but very few cracks.

Fig. 10 shows an example of the type of defect found in $\mathrm{NiTi}$, holes and inclusions rather than cracks.

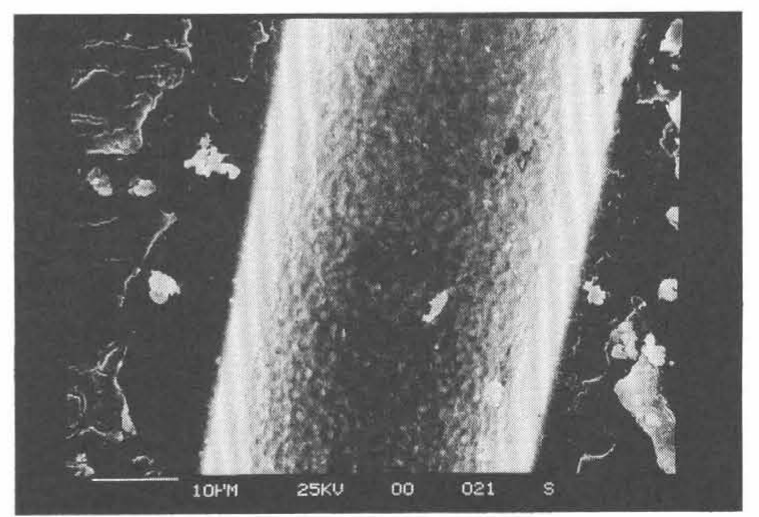

Fig.10 NiTi micro-EDMed sample 
Again, as with the control, the Ti sample showed no defects.

\section{CONCLUSIONS}

In general, with the stainless steels, the number of defects observed seemed to increase with the increasing Sulphur and Phosphorus contents (see Table 1). However, this does not explain why the NiTi sample should have been one of the poorestperforming materials of those tested. There appeared to be no correlation between the size of the defect (typically 2 to $10 \mu \mathrm{m}$ and larger in some cases) and the material composition. The most notable feature was the alignment of cracks in the rolling direction of the material.

The main conclusion to be drawn from this study is that it seems that most of the defects observed were inherent in the foil material before any machining had occurred, indicating material-dependence rather than machining-dependence. In addition, all the cracks observed in both the unmachined and machined samples were in alignment with the rolling direction. These foils undergo many rolling operations to reduce them to their final thickness. It has previously been noted that these microdefects, which are of little significance in macromachining, are highly significant in micromachining, implying the necessity for the development of defect-free multicrystalline "micromaterials" ${ }^{6)}$.

The size of these defects gives cause for concern, as they would certainly be sufficient to disrupt the flow of liquids should they occur on the bore of a nozzle. This is economically undesirable in the fabrication of ink jet nozzles, as the failure of just one jet in an array would reduce the performance of the whole nozzle leading to its rejection with all the consequences of manufacturing costs. It is suggested that the choice of a defect-free material for the printing head is therefore critical for economic manufacture.

In terms of the performance of the six samples tested as suitable nozzle materials a preliminary ranking order is:

$$
\begin{aligned}
& \text { Ti } \\
& \text { AISI } 316 \text { LS } \\
& \text { AISI } 304 \\
& \text { AISI } 316 \text { LN } \\
& \text { NiTi } \\
& \text { BS } 316 \text { S13 }
\end{aligned}
$$

Ti was the best nozzle material of those tested and BS 316 S13 the worst.
The aim of this particular study was to identify the presence of any defects. Consequently, all SEM micrographs taken were specifically of irregularities found and therefore not necessarily representative of the bulk material. Therefore, this highlighted the fact that defects do indeed occur but a more quantitative approach is now needed to determine the likelihood of machining a micro-hole in a "defect-containing" area given the specific array dimensions (hole size, number of holes and pitch). This can be achieved by using image-analysis techniques to analyse defects on a statistical basis and is in fact the subject of current ongoing research.

\section{ACKNOWLEDGEMENTS}

The authors would like to extend their grateful thanks to EPSRC for their support in providing funding for the micro-EDM centre at Cranfield.

\section{REFERENCES}

1. W. Ehrfeld, H. Lehr, F. Michel, A. Wolf, H.-P. Gruber and A. Bertholds, "Micro Electro Discharge Machining as a Technology in Micromachining," Proceedings of SPIE Symposium on Micromachining and Microfabrication, 2879, pp.332-337, 1996.

2. D.M. Allen and X.X. Huang, "AFM Quantitative Data Provides a New Understanding of Microelectro-Discharge Machined Surfaces," International Journal of Electrical Machining, 4, pp.7-12, 1999.

3. T. Masuzawa, C.-L. Kuo and M. Fujino, "A Combined Electrical Machining Process for Micronozzle Fabrication," Annals of the CIRP, 43, 1, pp. 189-192. 1994.

4. D.M. Allen and A. Lecheheb, "Micro Electrodischarge Machining of Ink Jet Nozzles: Optimum Selection of Material and Machining Parameters," Journal of Materials Processing Technology, 58, pp. 53-66, 1996.

5. A. Wolf, W. Ehrfeld, H. Lehr, F. Michel, M. Nienhaus and H.-P. Gruber, "Combining LIGA and Electro Discharge Machining for the Generation of Complex Micro Structures in Hard Materials," Proceedings of 9th International Precision Engineering Seminar and 4th Conference in Ultraprecision in Manufacturing Engineering, 2, pp. 657-660, 1997.

6. D. M. Allen, H. J. A. Almond, J. S. Bhogal, A. E. Green, P. M. Logan and X. X. Huang, "Typical metrology of micro-hole arrays made in stainless steel foils by two-stage micro-EDM," Annals of the CIRP, 48/1, pp. 127-130, 1999 